\title{
Application of ECH to the Study of Transport in ITER Baseline Scenario-like Discharges in DIII-D
}

\author{
R.I. Pinsker, a, M.E. Austin ${ }^{2}$, D.R. Ernst ${ }^{3}$, A.M. Garofalo ${ }^{1}$, B.A. Grierson ${ }^{4}$, J.C. Hosea ${ }^{4}$, T.C. Luce ${ }^{1}$, A. Marinoni ${ }^{3}$, \\ G.R. McKee ${ }^{5}$, R.J. Perkins ${ }^{4}$, C.C. Petty ${ }^{1}$, M. Porkolab ${ }^{3}$, J.C. Rost ${ }^{3}$, L. Schmitz ${ }^{6}$, W.M. Solomon ${ }^{4}$, G. Taylor ${ }^{4}$ \\ and F. Turco ${ }^{7}$
}

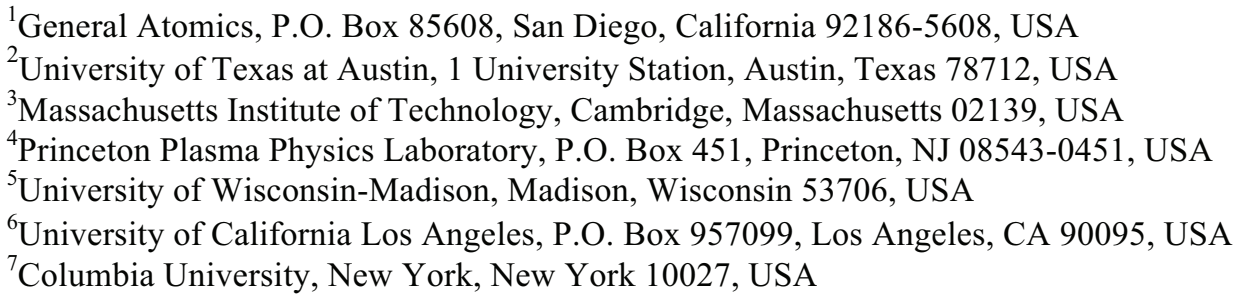

\begin{abstract}
Recent DIII-D experiments in the ITER Baseline Scenario (IBS) have shown strong increases in fluctuations and correlated reduction of confinement associated with entering the electron-heating-dominated regime with strong electron cyclotron heating $(\mathrm{ECH})$. The addition of $3.2 \mathrm{MW}$ of $110 \mathrm{GHz} \mathrm{EC}$ power deposited at $\rho \sim 0.42$ to IBS discharges with $\sim 3 \mathrm{MW}$ of neutral beam injection causes large increases in low-k and medium-k turbulent density fluctuations observed with Doppler backscatter (DBS), beam emission spectroscopy (BES) and phase-contrast imaging (PCI) diagnostics, correlated with decreases in the energy, particle, and momentum confinement times. Power balance calculations show the electron heat diffusivity $\chi_{\mathrm{e}}$ increases significantly in the mid-radius region $0.4<\rho<0.8$, which is roughly the same region where the DBS and BES diagnostics show the increases in turbulent density fluctuations. Confinement of angular momentum is also reduced during ECH. Studies with the TGYRO transport solver show that the model of turbulent transport embodied in the TGLF code quantitatively reproduces the measured transport in both the neutral beam (NB)-only and in the NB plus EC cases. A simple model of the decrease in toroidal rotation with EC power is set forth, which exhibits a bifurcation in the rotational state of the discharge.
\end{abstract}

\section{The ITER Baseline Scenario on DIII-D}

Properties of discharges in the baseline operating scenario for ITER [ITER-similar shape, $\beta_{\mathrm{N}} \sim 2, \mathrm{q}_{95} \sim 3$, ELMing $\mathrm{H}$-mode with $\left.\mathrm{H}_{98}(\mathrm{y}, 2) \sim 1\right]$ are the subject of ongoing experiments on DIII-D; recent work has extended the similarities to include ITER-relevant (low) torque and $T_{\mathrm{e}} / T_{\mathrm{i}} \sim 1$ (dominant electron heating). The aim of this work is to study confinement in the low rotation, dominant electron heating regime (as from alphas in a burning D-T plasma in ITER). The advantage of electron cyclotron heating as a principal heating tool for these studies is the fine control of the power deposition profile that is possible and the fact that all of the power goes to the electrons allows detailed study of the transport properties of the discharge.

In this paper, we compare ITER Baseline Scenario (IBS) discharges with neutral beam (NB) heating alone with otherwise identical discharges in which some of the
NB power is replaced with electron cyclotron heating $(\mathrm{ECH})(110 \mathrm{GHz} \mathrm{X} 2$, radial launch, deposition at $\rho \sim 0.45$ ). We examine the global confinement properties in the two cases, and analyze the radial profiles of diffusivities in both the electron and ion channels. Next, we carry out linear microstability analysis with the GS2 model to evaluate stability of ion temperature gradient (ITG) (long wavelength) and electron temperature gradient (ETG)/trapped electron modes (TEMs) (shorter wavelength), and compare the growth rates of the long wavelength modes with the measured shearing rates to estimate the effect of the $E_{\mathrm{r}}$ shear on the ITG modes. Using the TGYRO nonlinear transport solver we evaluate the turbulent transport model embodied in the TGLF code, and find that the measured profiles appear to be consistent with the predicted ones inside $\rho<0.8$ within the error bars, for both the NB-only and NB plus EC cases. A simple model for the important changes in toroidal rotation caused by $\mathrm{ECH}$ is described, which exhibits a

\footnotetext{
${ }^{\mathrm{a}}$ Corresponding author: pinsker@fusion.gat.com
} 
bifurcation into high and low rotation states of the discharge. Finally, we study the measured long- and intermediate-wavelength density fluctuations measured with the available diagnostics in both cases and find that the changes in the fluctuations appear to be qualitatively consistent with expectations derived from the modeling.

\section{Global confinement properties of IBS discharges with and without $\mathrm{ECH}$}

The time histories of the global parameters of the two comparison discharges $\left(\mathrm{I}_{\mathrm{p}}=1.3 \mathrm{MA}, \mathrm{B}_{\mathrm{T}}=1.75 \mathrm{~T}\right)$ are shown in Fig. 1. In both discharges, the neutral beam power is feedback controlled so that the stored energy and normalized beta, $\beta_{\mathrm{N}}$, follow a program, with the steady-state value of $\beta_{\mathrm{N}}=2.0$. In discharge 155199 [Fig. 1(b)], the $3.3 \mathrm{MW}$ of $110 \mathrm{GHz} \mathrm{ECH}$ is applied simultaneously with the feedback control of the NB power and the $\mathrm{ECH}$ power continues throughout the remainder of the discharge shown. Discharge 155196 [Fig. 1(a)] is identical up to time $2.5 \mathrm{~s}$, at which time the $\mathrm{ECH}$ power is turned off, and the programmed NB torque is reduced (by substituting counter-current injected power for some of the co-injected power). The torque was reduced to avoid an abrupt increase in co-rotation at the turn-off of the EC power.

A reduction in energy confinement with $\mathrm{ECH}$ is evident from the response of the feedback-controlled NB power at the turnoff of the ECH: though $3.3 \mathrm{MW}$ of heating power is removed from 155196 at $2.5 \mathrm{~s}$, the neutral beam power level increases by $<1 \mathrm{MW}$ to replace the EC power. Similarly, the reduced level of central toroidal rotation by $2.9 \mathrm{~s}$ (second timeslice shown on 155196, also shown on 155199) in the EC+NB case compared with the NB-only case, despite the higher level of applied torque in the former case, is evidence of a significant reduction in toroidal momentum confinement time with EC. Also, the higher line-averaged density observed in the NB-only case by $2.9 \mathrm{~s}\left(5.7 \times 10^{19} \mathrm{~m}^{-3}\right)$ occurs despite the lack of gas puffing (not shown in Fig. 1) in that case, while to maintain a lower, programmed value of density of $5.0 \times 10^{19} \mathrm{~m}^{-3}$ in the $\mathrm{EC}+\mathrm{NB}$ case requires significant (feedback-controlled) puffing - evidence of the familiar density "pumpout" effect with EC, e.g. a reduction in global particle confinement time often associated with ECH [1].

However, there is no reason to believe that the lower confinement in the $\mathrm{EC}+\mathrm{NB}$ case compared to the lowerpower NB-only case is a result of some rf-specific effect. Rather, this effect results from incremental torque-free direct electron heating. In fact, the small decrease in thermal stored energy upon turning off the ECH is largely explained by the strong power degradation in the $\mathrm{H}$-mode confinement scaling relation. This can be quantified by looking at energy confinement a few $\tau_{\mathrm{E}}$ after the EC turnoff and comparing with the same time in the discharge with continued EC, as follows.

Since the toroidal rotation begins to slow in the lowtorque, NB-only case after about $2.9 \mathrm{~s}$, eventually leading

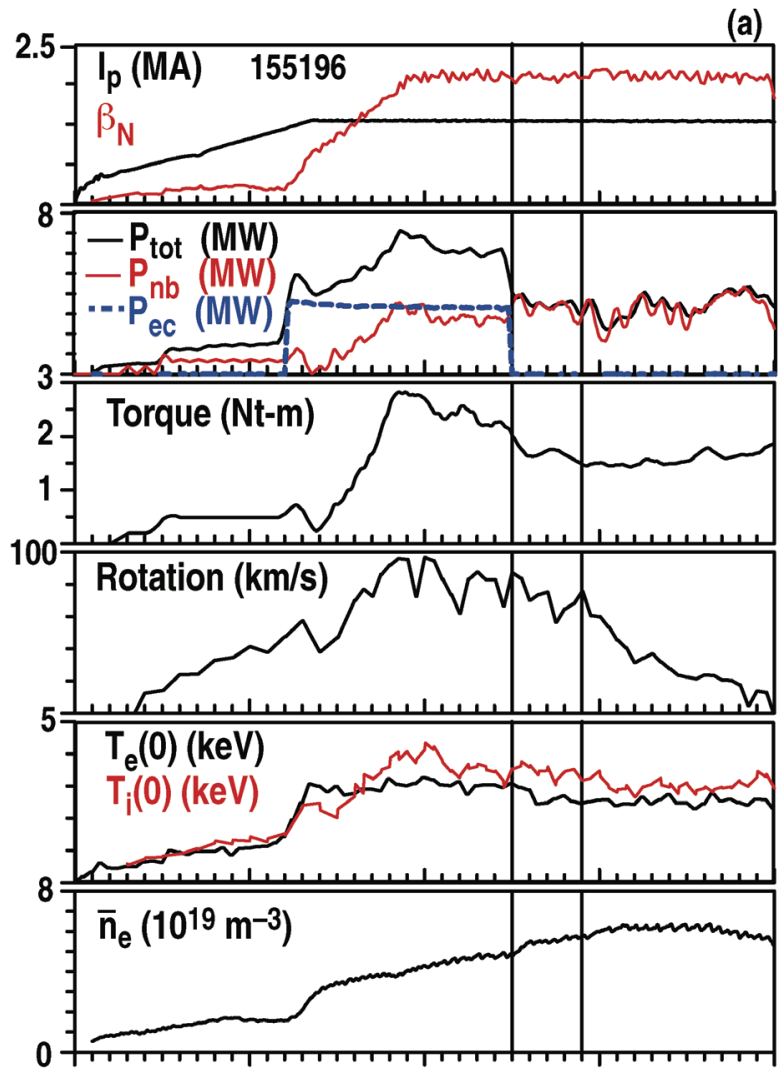

(a)

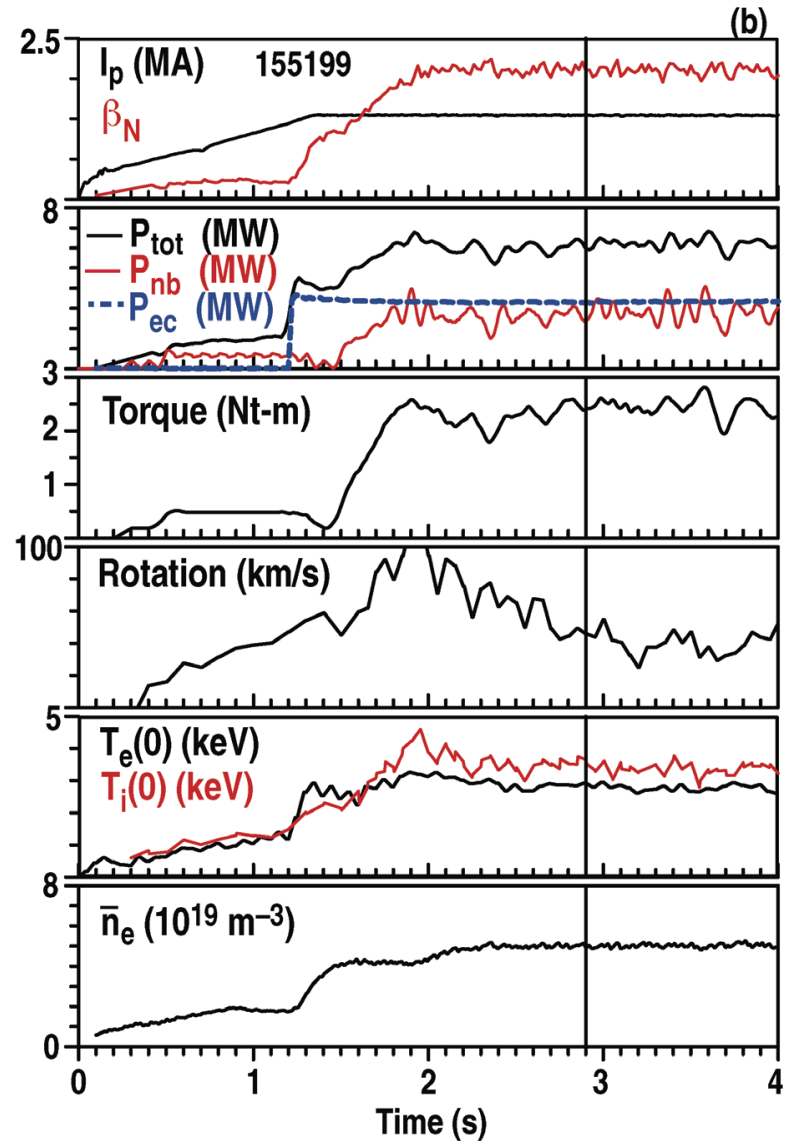

Figure 1. Time histories of global parameters of discharges (a) with pure NB heating after $2.5 \mathrm{~s}$ and (b) NB plus ECH throughout, at a fixed stored energy and beta in the quasisteady-state portion of the discharge. 
to a locked mode and degraded confinement at late times (a phenomenon which was observed in virtually every low-torque discharge in this series, and is a topic of ongoing study), we quantify the energy confinement comparison at $2.9 \mathrm{~s}$ from TRANSP [2] calculations of the thermal stored energy in Table 1.

Table 1. TRANSP calculations of stored energy, confinement time, and comparison with IPB98 $(\mathrm{y}, 2)$ scaling for the EC+NB (155199) and NB-only case (155196).

\begin{tabular}{|c|c|c|}
\hline & $\begin{array}{l}155199 \\
\text { at } 2.9 \mathrm{~s}\end{array}$ & $\begin{array}{l}155196 \\
\text { at } 2.9 \mathrm{~s}\end{array}$ \\
\hline$P_{\mathrm{NB}}-P_{\text {losses }}$ & $2.6 \mathrm{MW}$ & $2.7 \mathrm{MW}$ \\
\hline$P_{\mathrm{OH}}$ & $0.2 \mathrm{MW}$ & $0.3 \mathrm{MW}$ \\
\hline$P_{\mathrm{EC}}$ & $\underline{3.3 \mathrm{MW}}$ & $\underline{0.0 \mathrm{MW}}$ \\
\hline$P_{\text {tot }}$ & $6.1 \mathrm{MW}$ & $3.0 \mathrm{MW}$ \\
\hline$W_{\text {tot }}$ & $0.74 \mathrm{MJ}$ & $0.715 \mathrm{MJ}$ \\
\hline$W_{\mathrm{th}}$ & $0.68 \mathrm{MJ}$ & $0.665 \mathrm{MJ}$ \\
\hline$\tau_{\mathrm{E}, \mathrm{th}}$ & $0.111 \mathrm{~s}$ & $0.222 \mathrm{~s}$ \\
\hline$\tau_{\mathrm{E}, \mathrm{th}}[\mathrm{IPB} 98(\mathrm{y}, 2)]$ & $0.126 \mathrm{~s}$ & $0.217 \mathrm{~s}$ \\
\hline $\mathrm{H} 98(\mathrm{y}, 2)$ & 0.88 & 1.02 \\
\hline
\end{tabular}

Because of the expected thermal energy confinement time scaling with total power and line-averaged density is as $\tau_{\mathrm{E}, \mathrm{h}} \sim \mathrm{P}^{-0.69} \mathrm{n}_{\mathrm{e}}^{0.41}$, the thermal stored energy would be expected to increase with ECH from 0.665 MJ (155196) by a factor of only $(6.1 / 3.0)(6.1 / 3.0)^{-0.69}(5.0 / 5.7)^{0.41}=$ $(2.03)^{+0.31}(0.88)^{0.41}=1.18$, to $0.79 \mathrm{MJ}$, despite the more than factor-of-two increase in heating power. The slightly smaller increase observed, to $0.68 \mathrm{MJ}$, can be understood through changes in the local heat transport as discussed in Sec. 3 .

We conclude from this examination of the global behavior of confinement that the results appear to be approximately consistent with standard $\mathrm{H}$-mode scaling, with or without EC power. The advantage of studying the comparison in more detail, extending the 0D approach to 1D (radial profiles), is that the case with ECH has a very well-characterized heat source, so that quantitative calculations of diffusivities can be performed and compared with theory-based expectations.

\section{Profiles, diffusivities, and comparison with turbulent transport models}

The diffusivities are calculated from the measured profiles in TRANSP in conjunction with models for the neutral beam and ECH power deposition profiles. The profiles of electron and ion temperature, electron density and toroidal rotation are shown in Fig. 2 for the two discharges at $2.9 \mathrm{~s}$, with the profiles from the case with NB only as dashed curves. The electron density near the magnetic axis is derived from the frequency of the first electron cyclotron emission (ECE) channel that is cutoff (one cutoff point is visible below the electron temperature profile curve); this measurement is independent of the Thomson scattering density calibration. The addition of $\mathrm{ECH}$ exchanges increased electron temperature for a decreased electron density across the profile. Perhaps the most striking effect of the $\mathrm{ECH}$ is to reduce the toroidal rotation speed and its gradient (velocity shear) significantly in most of the core of the plasma.

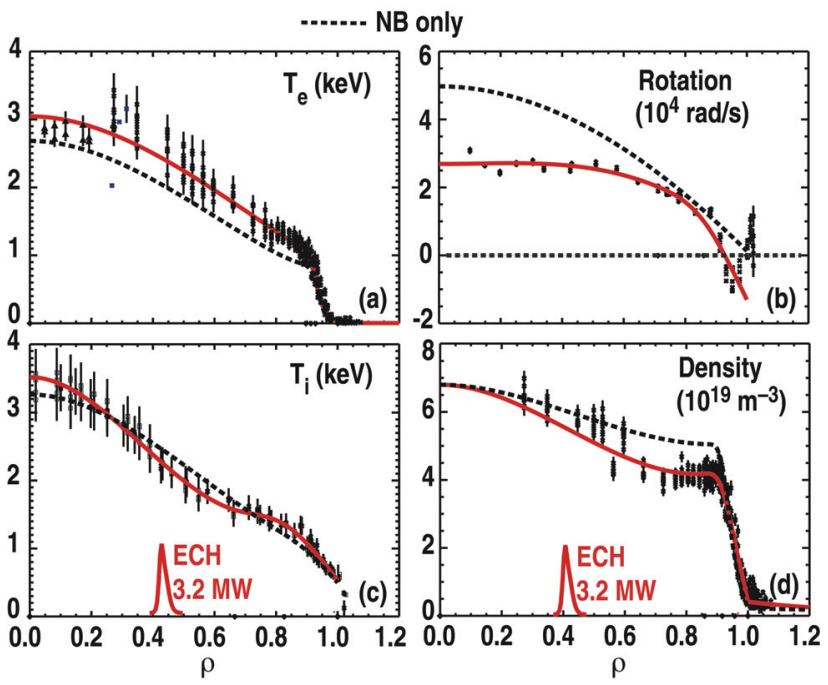

Figure 2. Profiles of electron and ion temperature, electron density, and toroidal rotation for the two discharges at $2.9 \mathrm{~s}$, with the profiles from the case with NB only as dashed curves. The radial location and profile shape of the ECH deposition is shown as the small curve along the radial axis.

The ion and electron heat diffusivities calculated by TRANSP agree with those calculated by ONETWO, and are exhibited in Fig. 3 with error estimates based on Monte-Carlo error propagation of the experimental measurements. The paucity of profile data near the magnetic axis leads to large error bars on the diffusivities in that region, but in these sawtoothing discharges, it would be difficult to draw conclusions about transport in that region in any event. Both electron and ion heat diffusivities are significantly larger with $\mathrm{ECH}$ in the midradius region, with the increases in that region being well outside the error bars.

In order to compare these results with the models embodied in codes such as TGLF (a gyrofluid model) [3] and GYRO [4] or GS2 [5] (gyrokinetic models), a first step is to compute the linear growth rates of the dominant drift-wave instabilities and compare the growth rates of the relatively long-wavelength ITG modes with the ExB shearing rate in accordance with the "quench rule" [6]. We have carried out linear runs of the GS2 code using the measured profiles from the two discharges studied here, with the resulting ITG growth rates at the poloidal wavenumber at which the growth rate is maximized displayed in Fig. 4. The growth rate is maximum at a normalized poloidal wave number of about $k_{\theta} \rho_{i} \sim 0.6$. Also shown are the ExB flow shearing rates estimated from the Hahm-Burrell formula [7]. In the present case, the radial electric field is dominated by the toroidal flow velocity crossed into the poloidal magnetic field; the terms related to the (small) poloidal flow and the ion pressure gradient term are negligible in the mid-radius region of interest here. The usual estimate of the effect of the ExB flow shear is that if the shearing rate is larger than the growth rate, the turbulence will be quenched $[7,8]$. Although the somewhat larger density gradient in 
the mid-radius region in the $\mathrm{EC}+\mathrm{NB}$ case (resulting from the reduction in the density at the top of the pedestal from EC "pumpout") reduces the growth rate of the linear ITG slightly, the lower toroidal flow velocity and reduced shear in that case leads to a prediction of substantially larger saturated fluctuation amplitudes in the region of $0.4<\rho<0.65$ compared with the NB-only case. In the latter case, the estimate would lead to the expectation that the ITG turbulence is quenched at all radii by the flow shear. Reference 8 warns that the "quench rule" is only approximate; computationally much more expensive nonlinear calculations should be carried out for this case to verify this conclusion.
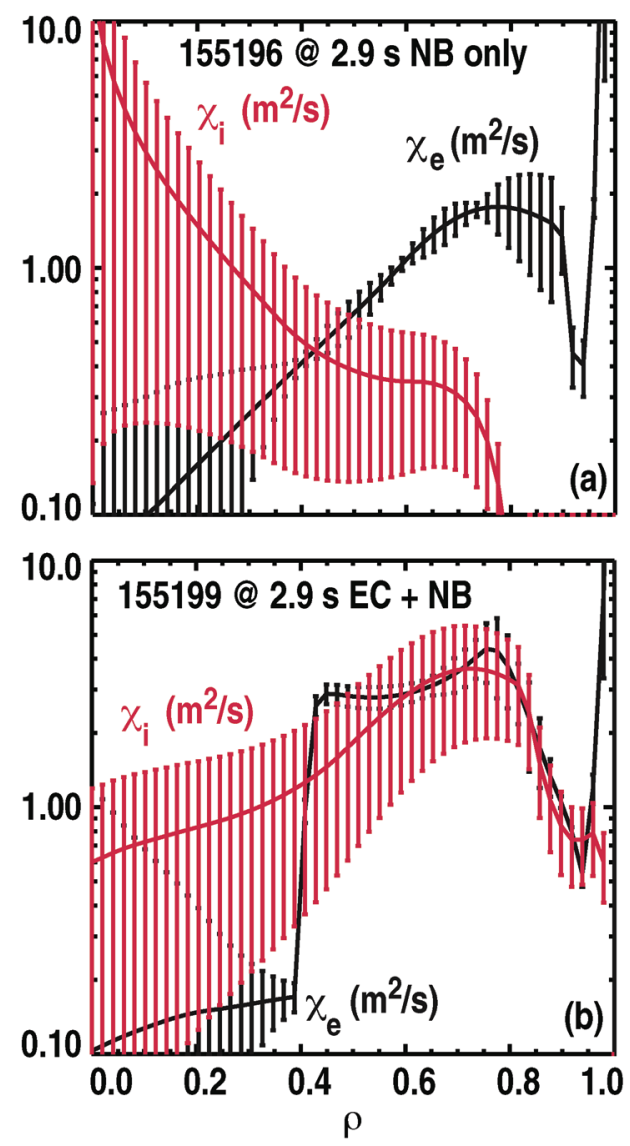

Figure 3. Deduced ion and electron heat diffusivities from TRANSP for NB-only (a) and NB+ECH (a) cases.

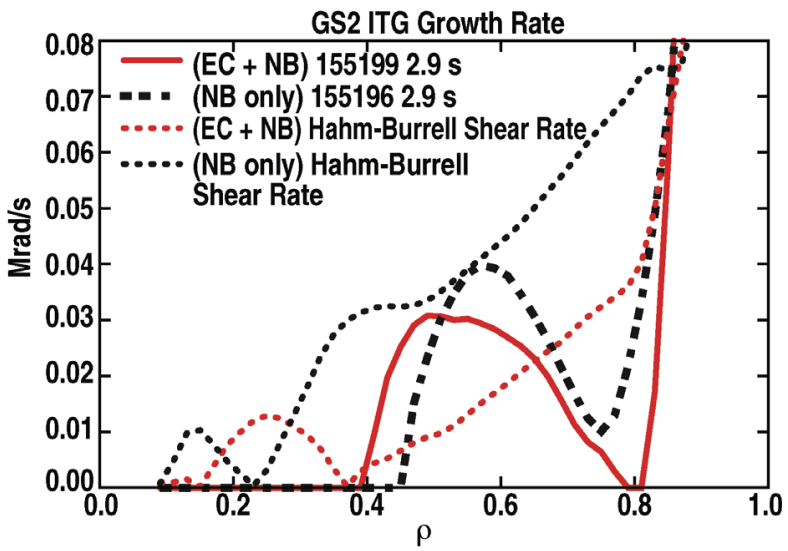

Figure 4. GS2 calculations of the growth rate of the most unstable ITG modes compared with the Hahm-Burrell estimate of the shearing rate for the $\mathrm{EC}+\mathrm{NB}$ case and the NB-only case.

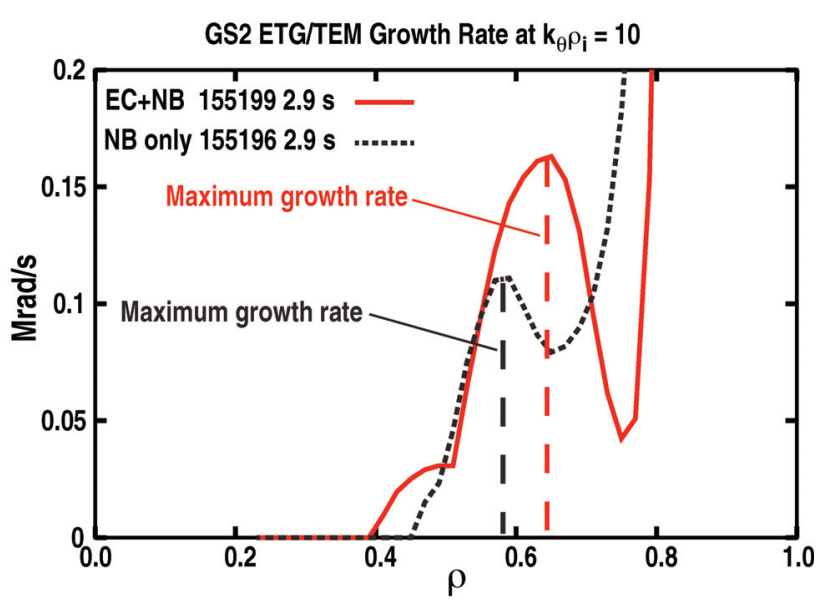

Figure 5. GS2 calculations of the growth rate of the ETG/TEM modes for the $\mathrm{EC}+\mathrm{NB}$ case and the NB-only case.

We have also calculated the linear growth rates of the shorter wavelength modes at $k_{\theta} \rho_{i}=10$, in the ETG/TEM range (Fig. 5). These short wavelength modes are not significantly affected by the large-scale flow shear. The growth rates are higher by $\sim 50 \%$ in the radial zone $0.55<\rho<0.7$ for the $\mathrm{EC}+\mathrm{NB}$ case compared to the NB-only case, due to the higher value of $T_{\mathrm{e}} / T_{\mathrm{i}}$ in that region. Whether or not these electron modes have an important effect on electron heat transport cannot be determined from these linear calculations alone; nonlinear calculations are necessary to ascertain whether there is a cascading to longer wavelengths that might render turbulence due to these modes observable with the available fluctuation diagnostics on DIII-D (see below).

For trapped electron modes, gyrokinetic codes like GS2 are more accurate than the gyrofluid model TGLF. However, while it is practical to perform linear stability analysis with GS2 as shown above, evolving profiles on transport timescales would require multiscale gyrokinetic transport simulations, which although possible, require resources beyond those available for this work. Therefore we have used the reduced model Trapped Gyro-Landau Fluid (TGLF), which uses saturation rules calibrated from a database of nonlinear GYRO simulations [3] to evolve profiles on transport timescales, called from the TGYRO transport solver [9]. Stiff turbulent transport is extremely sensitive to the driving gradient, by definition, so MonteCarlo propagation of electron and ion temperature profile gradients through TGLF will produce a very large range of possible energy fluxes. TGYRO solves the inverse problem by determining the local gradient scale lengths that produce an energy flux that matches the power balance flux (from TRANSP) in an iterative manner. Profiles of $T_{\mathrm{e}}$ and $T_{\mathrm{i}}$ are constructed by integrating inwards from a "fixed point" on the profile, here chosen at $\rho=0.8$, determining the profile for all points at smaller minor radius than the fixed point. A transport solution is obtained when the TGLF flux matches the power balance flux, as displayed in Fig. 6(a,c), with the reconstructed profiles displayed in Fig. 6(b,d). In the results reported here, the energy exchange between electrons and ions is recomputed in each iteration making the power flow selfconsistent with the profile prediction. From the TGYRO modeling of these plasmas, the electron transport is 
strongly dominated by turbulence in both discharges with negligible neoclassical transport (ion transport has a larger neoclassical contribution). The stiff nature of the transport in these plasmas is manifested by the modest increase in the electron temperature profile gradient at $\rho=0.45$ to produce approximately seven times the electron energy flux. Transport solution profiles obtained from TGYRO with NEO and TGLF are within the experimental measurement errors. In both cases the ion transport is also well accounted for by NEO and TGLF, although in the discharge with EC heating there is a significant increase in the turbulent contributions to the ion energy flux. In the beam heated plasma at mid radius approximately $60 \%$ of the ion energy flux is from turbulent transport, but in the EC+NB plasma with lower toroidal rotation approximately $80 \%$ of the ion energy transport comes from turbulence. Angular momentum transport in TGYRO is in a testing phase and therefore predictions of the toroidal rotation are not included here. However it is noteworthy that when the flux-matching gradients of $a / L_{\mathrm{Te}}, a / L_{\mathrm{Ti}}$ and $a / L_{\mathrm{ne}}$ are obtained with TGYRO the TGLF momentum flux is qualitatively similar to the toroidal angular momentum flux from TRANSP in both beam-heated and beam+EC-heated plasmas. These discharges are the subject of ongoing transport model validation studies in all (energy, particle and momentum) channels and will be reported in future work.
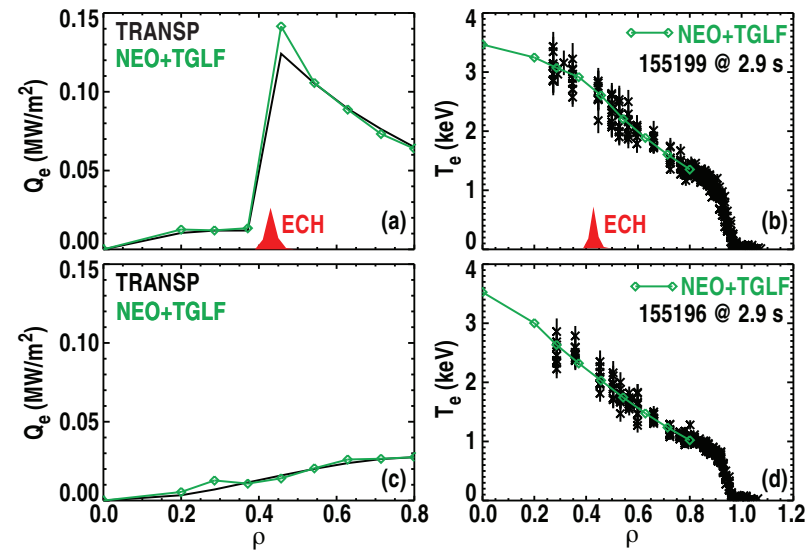

Figure 6. Results from TGYRO nonlinear fitting algorithm for discharge with $\mathrm{EC}+\mathrm{NB}(\mathrm{a}, \mathrm{b})$ and $\mathrm{NB}$ only $(\mathrm{c}, \mathrm{d})$, demonstrating that the TGLF model provides a good quantitative fit to the data in both cases within the error bars in the mid-radius region.

\section{Simple bifurcation model for toroidal rotation slow-down with $\mathrm{ECH}$}

We may conclude that the measured profiles, including rotation, in cases with and without EC are consistent with the quasi-linear model of drift-wave turbulent transport implemented in TGLF. The GS2 calculation showed that the crucial difference between the case with EC from without is the reduced toroidal rotation and its shear. What this does not address is why the EC case has such significantly reduced rotation. In previous work, such a reduction in rotation has been observed due to incremental electron heating with fast waves or ECH in DIII-D [10], or minority ion cyclotron resonance heating (ICRH) in AUG [11], for example, and in those cases the effect was attributed to a link between momentum diffusivity $\chi_{\phi}$ and ion heat diffusivity $\chi_{i}$. In the present case, it is apparent that the increase in momentum diffusivity is significantly larger than the increase in the ion heat diffusivity, i.e., the ratio $\chi_{\phi} / \chi_{i}$ (the Prandtl number) increases with the addition of ECH. TRANSP analysis of the experimental case (not shown here) suggests such an increase in Prandtl number in the present case. Based on the results of gyrokinetic simulations of momentum transport, we develop a simple model to illustrate that a bifurcation from a state of higher toroidal rotation without $\mathrm{ECH}$ to one of lower toroidal rotation during ECH would be consistent with our results.

The experimental data in this case has too low time resolution to accurately track the evolution of the rotation at ECH turnoff; the goal of the following calculation is to illustrate that the momentum balance combined with a model of the results of nonlinear gyrokinetic computations in the published literature yield a prediction of two different self-consistent quasi-steady rotational states of the plasma with the same applied torque. To determine whether or not this bifurcation of the rotation and its shear describes what actually happens in the experiment will require future experimental and theoretical work: further experiments optimized to examine the dynamics of the changes in rotation with $\mathrm{ECH}$ are needed, and development of the model to calculate those dynamic aspects will be necessary.

The angular momentum balance equation with the neutral beam torque density $S$ may be written as

$$
\frac{1}{r} \frac{\partial}{\partial r}\left(r m n R^{2} \chi_{\phi}^{\text {eff }} \frac{\partial V_{\phi} / R}{\partial r}\right)+S=0 \quad,
$$

in which $r$ is the minor radius, $m n$ is the mass density, $R$ is the major radius, $V_{\phi}$ is the toroidal velocity, and $\chi_{\phi}^{e f f}$ is the total effective momentum diffusivity, which for our purposes originates from the symmetry breaking due to shear in the parallel and ExB flows only. This "high flow" ordering, while appropriate for the strongly rotating cases described here (see, e.g. Ref. [12]), does not include higher order terms in gyroradius that may be required to consistently describe diamagnetic-level flows. However, the consistency with TGLF, including matching the radial profile of the residual stress, suggests an accurate calculation of diamagnetic-level toroidal flows is not an essential ingredient in these cases.

Since in this case the poloidal rotation and pressure gradient terms in the radial force balance are small relative to the toroidal velocity term, the radial electric field can be approximated as $E_{r} \approx V_{\phi} B_{\theta}$, where $B_{\theta}$ is the poloidal magnetic field. The ExB shear rate is then $\gamma_{E}=(\varepsilon / q)\left|\partial V_{\phi} / \partial r\right|$, where $\varepsilon=r / R$ is the inverse aspect ratio, and $q$ is the safety factor. Because the beam torque density changes little upon injection of ECH, Eq. (1) yields a constraint on the radial flux of toroidal momentum: 


$$
\chi_{\phi}^{e f f} \gamma_{E}=\frac{1}{m n q R^{2}} \int_{0}^{r} d r^{\prime} r^{\prime} S\left(r^{\prime}\right) .
$$

The dominant symmetry breaking terms contributing to the momentum diffusivity are the parallel and perpendicular flow shears, $u_{\|}^{\prime}$ and $\gamma_{\mathrm{E}}$, whose separate roles were studied in Ref. [13]. In the case of purely toroidal flow, $u_{\|}=\left(B_{\phi} / B\right) \Omega R$, where $\Omega R=V_{\phi}$, so the two terms are constrained to vary together by $u_{\|}^{\prime} \approx(q / \varepsilon) \gamma_{E}$. The dependence of the effective Prandtl number $\chi_{\phi}^{\text {eff }} / \chi_{i}$ on $u_{\|}^{\prime}\left(a / c_{s}\right)$, for purely toroidal flow, where $\chi_{i}$ is the ion thermal diffusivity, $a$ is the minor radius, and $c_{s}$ is the sound speed, has been investigated with flux-tube nonlinear gyrokinetic simulations in Ref. [14], and the relevant results are shown in Fig. 14 of that work. We have fit an analytic function $f\left(u_{\|}^{\prime} a / c_{s}\right)$ to their results for the case $\varepsilon / q \approx 0.1$ and $\gamma_{\max }=0.2 c_{s} / a$, shown in Fig. 7(a). The reduced Prandtl number at smaller toroidal rotation shear is due to the effect of the ExB shear symmetry-breaking term, which acts against the parallel flow shear term [14]. While some details may differ in our specific case relative to Ref. [14], the important point is that the dependence of Prandtl number on flow shear is rather weak in the case of purely toroidal flow. In fact, the details of this dependence are unimportant for our purposes, and it will be sufficient to approximate $f \approx 0.65$. To determine the full dependence of momentum transport on toroidal flow shear, we now invoke the quench rule for the ion thermal diffusivity [6] to write $\chi_{i}=\chi_{i 0}\left(1-\alpha_{E} \gamma_{E} / \gamma_{\max }\right)$, in which $\alpha_{\mathrm{E}}=0.5$ approximately reproduces nonlinear GYRO results [15]. This yields the expression for variation of the normalized radial flux of toroidal momentum on the left hand side of Eq. (2). Figure 7(b) compares the detailed dependence of $f$ with the approximation $f \approx 0.65$, showing the detailed dependence of $f$ is unimportant. Inserting this into Eq. (2) yields a quadratic equation for the shearing rate, $\zeta=\gamma_{E} / \gamma_{\max }$,

$$
\left(1-\alpha_{E} \zeta\right) \zeta f=\frac{\left(\chi_{i 0} \gamma_{\max }\right)^{-1}}{m n q R^{2}} \int_{0}^{r} d r^{\prime} r^{\prime} S\left(r^{\prime}\right) \equiv \eta_{0},
$$

which may be solved for two rotation states. As shown in Fig. 4, the ITG growth rate does not change appreciably with the application of ECH, so we expect $\chi_{i 0}$ and therefore $\eta_{0}$ to remain relatively constant. Solving Eq. (3) for $\xi$ yields two solutions, showing that a bifurcation from a high rotation state to a low rotation state is possible, as illustrated in Fig. 7(b). The two solutions of Eq. (3) are $\xi=1 \pm\left(1-2 \eta_{0} f^{-1}\right)^{1 / 2}$, where we have taken $\alpha_{E}=0.5$. The dynamics of this possible bifurcation remain to be explored, specifically how the forbidden higher momentum flux region between the two solutions is crossed to go from high rotation to low rotation. Further, the linear dependence of momentum diffusivity on flow shear that we have utilized in this illustration remains valid only for shearing rates much smaller than growth rates [12]. The variation of momentum diffusivity with flow shear is expected to be significantly nonlinear in these cases, but the qualitative features we describe should remain.
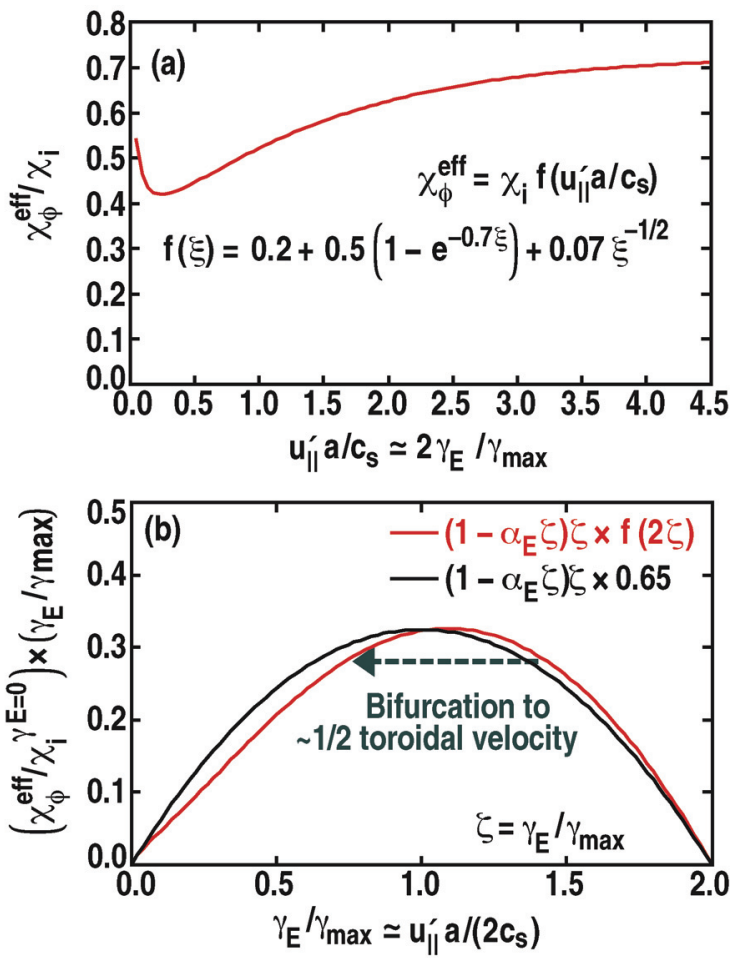

Figure 7. (a) Fit to gyrokinetic simulation results of Casson et al. (Ref. [14], Fig. 14) for the effective Prandtl number in the case of pure toroidal rotation, for $\varepsilon / q=0.1$. (b) Dependence of normalized radial flux of toroidal momentum on flow shear, assuming purely toroidal rotation and linearized transport. Comparison with a simple model for $\chi_{\phi}^{\text {eff }}$, which is linear in the flow shear, shows the details of the function $f\left(u_{\|}^{\prime} a / c_{\mathrm{s}}\right)$ are relatively unimportant. For fixed torque (fixed momentum flux), two values of flow shear are possible, showing that a bifurcation from large to small values of centrally peaked toroidal rotation can occur. A strong increase in effective Prandtl number would accompany the slowdown.

\section{Effects on observed density fluctuations}

One of the important goals of the experiments discussed here was to measure the direct effect of the incremental electron heating on the turbulent density fluctuations measured in the core with all of the available diagnostics. These fluctuation diagnostics include Beam Emission Spectroscopy (BES) to look at long wavelength (in the ITG range) turbulence [16], Doppler Backscattering (DBS) looking at intermediate wavelength modes by a technique closely related to reflectometry [17], and phase-contrast imaging (PCI), examining chord-averaged fluctuations in a broad range of wavenumbers and a wide frequency range [18]. The fluctuations in the NB-only case are compared with those observed in the $\mathrm{EC}+\mathrm{NB}$ case, with each diagnostic.

The BES system observed a very striking difference in the fluctuations in the ITG range for the two cases 
(Fig. 8), with the fluctuation amplitude in the NB-only case being indistinguishable from the noise and quite clearly observable in the case with EC+NB. The total spectrally-integrated power is at least 16 times higher in the case with EC+NB. Very similar results were obtained on another shot-to-shot comparison from another run day. The setup of the instrument was such that it was sensitive to perpendicular wavenumbers $k_{\perp}<3 \mathrm{~cm}^{-1}$. A qualitatively similar result was obtained with the DBS system, shown in Fig. 9. The largest difference between the fluctuation levels with and without EC is observed in $0.4<\rho<0.5$ (i.e., the region in which the EC power is deposited), while the highest fluctuation amplitudes are observed in the EC+NB case at $\rho \sim 0.6$. The scattering geometry for the DBS is such that the wavenumbers being probed have $k_{\theta} \sim 6 \mathrm{~cm}^{-1}$ for $\rho \sim 0.6\left(k_{\theta} \rho_{\mathrm{s}} \sim 2\right)$, and $k_{\theta} \sim 8 \mathrm{~cm}^{-1}$ for $\rho<0.4\left(k_{\theta} \rho_{\mathrm{s}} \sim 3\right)$. Here, $\rho_{\mathrm{s}}$ is the ion sound Larmor radius $\rho_{\mathrm{s}}=\left(\kappa T_{e} M_{i}\right)^{1 / 2} / e B$. The probed wavenumber and the probed (cutoff) radius are determined via GENRAY ray tracing. The DBS poloidal wavenumber resolution is $\Delta k_{\theta} / k_{\theta} \sim 0.4-0.6$.

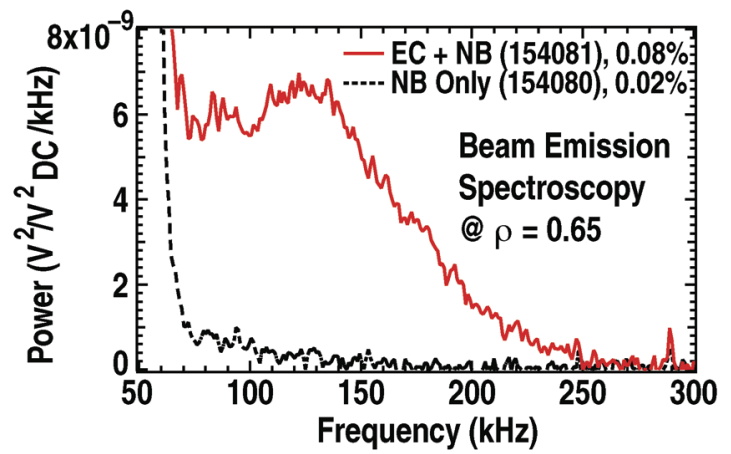

Figure 8. Beam emission spectroscopy measurements of density fluctuations at $k_{\perp}<3 \mathrm{~cm}^{-1}$ in an $\mathrm{EC}+\mathrm{NB} / \mathrm{NB}$-only comparison similar to the pair analyzed earlier.

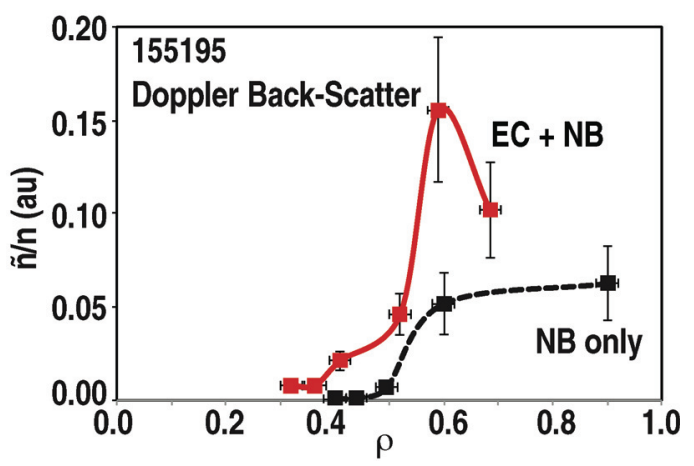

Figure 9. Density fluctuations measured with DBS from a single discharge, comparing a period with $\mathrm{NB}$ only with a period with $\mathrm{EC}+\mathrm{NB}$.

The fluctuations measured with PCI are also significantly higher in amplitude with $\mathrm{EC}+\mathrm{NB}$ than with NB alone (Fig. 10), at frequencies below $\sim 100 \mathrm{kHz}$, as shown in the inset (vertical scale linear in power, $10 \mathrm{kHz}<\mathrm{f}<100 \mathrm{kHz}$ ). However, as can be seen with a logarithmic power scale, the fluctuations at a few hundred $\mathrm{kHz}$ are actually somewhat higher with NB alone than with EC+NB. This effect is observed consistently with this instrument. The PCI measures fluctuations in a broad range of horizontally propagating wavenumbers from $1-30 \mathrm{~cm}^{-1}$, which along the vertical line integral are a combination of $k_{\theta}$ and $k_{\rho}$. It is possible that the frequency dependence of the signal may be related to the nonlinear behavior of the (saturated) drift wave turbulence.

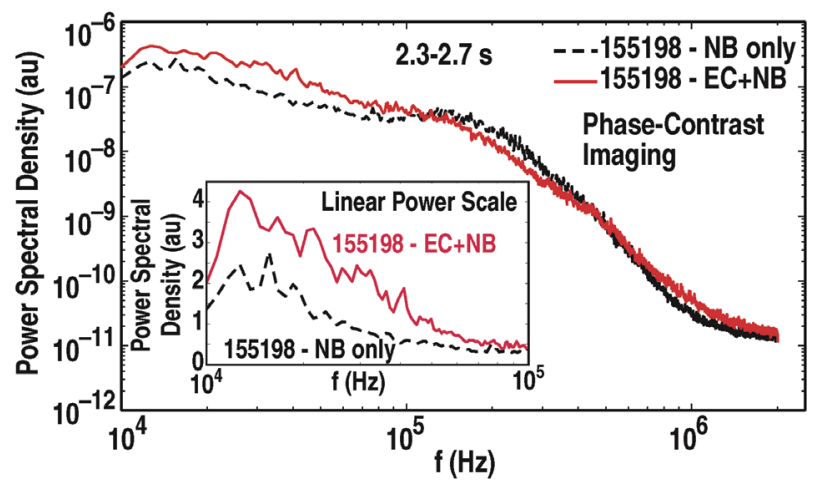

Figure 10. Density fluctuations averaged over a vertical chord at $\rho>0.4$ integrated over a broad range of radial wavenumbers measured with PCI, comparing a period with NB only with a period with $\mathrm{NB}+\mathrm{EC}$. The results are shown both on a linear power scale (inset) and on a logarithmic power scale over a broader frequency and amplitude range.

Since the PCI diagnostic does not need to integrate its signal over many energy confinement times in order to increase the signal-to-noise-ratio to acceptable levels, it was also used to compare the spectrum just before and after the EC shut-off in these cases to the spectrum obtained a few energy confinement times later. As expected, the low-frequency turbulence does not appear to change abruptly upon turning off the EC power; the characteristic increase in the amplitude of the lowfrequency turbulence occurs only after the profiles change.

The fluctuation diagnostics, then, show changes in the turbulent density fluctuations that are qualitatively consistent with expectations based on the most commonly used quasilinear model of turbulent transport. To make the comparison between the model and the measurements quantitative, it will be necessary to carry out simulations incorporating "synthetic diagnostics," i.e. models of what is actually measured with each particular diagnostic.

\section{Summary and conclusions}

We have studied in detail the properties of a pair of DIII-D discharges in the IBS regime, both of which were heated with NB throughout. In one case, ECH was also used throughout the quasi-steady portion of the discharge, while in the other case the ECH was turned off at $2.5 \mathrm{~s}$. Examining the global energy confinement properties at a time a few energy confinement times after the ECH turnoff, we find that both cases are roughly in accordance with IPB98 $(y, 2)$ confinement scaling, though the confinement time in the ECH case appears to scale more strongly with density than the scaling predicts. In more detail, the radial profiles in the core were shown to be 
accurately predicted in both cases by the TGLF model of turbulent transport. A key difference between the two cases is the slow-down in toroidal rotation and the reduction in the flow shear in the core caused by ECH; a simple model illustrates the possibility of a bifurcation in the rotation resulting from the increases in momentum diffusivity associated with the ECH and the nonlinear connection between the saturated turbulence amplitude and the flow shear. Three diagnostics were used to characterize the differences between the turbulent density fluctuations in the two cases, and each diagnostic documented differences that appear to be qualitatively consistent with the turbulent transport model.

Future work will attempt to quantify some of these qualitative results, by implementing synthetic versions of the fluctuation diagnostics in the computational turbulent transport model. The simplified model of the effect of $\mathrm{ECH}$ on rotation will be further developed and compared to the observed dynamics of the rotation upon sudden changes in the EC power; the present work considered only the quasi-steady conditions obtained after a few transport times had elapsed since the step-down in EC power. The fact that no significant discrepancies between the most commonly used model of turbulent transport have been found so far in these IBS studies, whether the heating is provided purely by $\mathrm{NB}$ or by $\mathrm{EC}+\mathrm{NB}$, is encouraging, in that a predictive model appears to be in hand which can be used to predict ITER's confinement in a low-torque, dominantly electron-heated regime, though the important topic of rotation and flow shear in lowtorque regimes, dominated by the properties of intrinsic rotation, remains for continued study. The companion to the NB-only and EC+NB discharges, which would be the EC-only case, is challenging to study in DIII-D, due to the important diagnostics that are based on the neutral beams (e.g., BES for the long-wavelength density fluctuations and charge-exchange recombination spectroscopy for ion temperature, flows, impurity density). However, EC-dominated cases with balanced beam injection (no net torque but non-negligible flow shear) will be studied in the near future. A long-term goal of this work is to use this understanding to find methods of improving confinement in this reactor-relevant regime.

\section{Acknowledgement}

This work supported by the US Department of Energy under DE-FC02-04ER54698, DE-FC02-08ER54966, DE-
AC03-09CH11466, DE-FG02-04ER54235, DEFG0289ER53296, DE-FG02-08ER54999, DE-FG0208ER54984, and DE-FG02-04ER54461.

\section{References}

1. V. Erckmann and U. Gasparino, Plasma Phys. Control. Fusion 36, 1869 (1994)

2. R. J. Hawryluk, et al., "An empirical approach to tokamak transport," in Physics of Plasmas Close to Thermonuclear Conditions, vol. 1, ed. B. Coppi, et al. (Brussels: CEC) p. 19 (1980)

3. G. M. Staebler, et al., Phys. Plasmas 21, 055902 (2014)

4. J. Candy and R. E. Waltz, J. Comput. Phys. 186, 545 (2003)

5. M. Kotschenreuther, et al., Comput. Phys. Commun. 88, 128 (1995)

6. R. E. Waltz, R. L. Dewar and X. Garbet, Phys. Plasmas 5, 1784 (1998)

7. T. S. Hahm and K. H. Burrell, Phys. Plasmas 2, 1648 (1995)

8. K.H. Burrell, Phys. Plasmas 4, 1499 (1997)

9. J. Candy, et al., Phys. Plasmas 16, 060704 (2009)

10. J. S. deGrassie, et al., Proc. 26th EPS Conf. on Controlled Fusion and Plasma Physics (Maastricht, 1999) vol. 23J, 1189 (1999)

11. D. Nishijima, et al., Plasma Phys. Control. Fusion 47, 89 (2005)

12. A. G. Peeters, et al., Nucl. Fusion 51, 094027 (2011)

13. R. E. Waltz, G. M. Staebler, J. Candy, and F. L. Hinton, Phys. Plasmas 14,122507 (2007)

14. F. J. Casson, et al., Phys. Plasmas 16, 092303 (2009)

15. J. E. Kinsey, R. E. Waltz, and J. Candy, Phys. Plasmas 12, 062302 (2005)

16. G. R. McKee, et al., Rev. Sci. Instrum. 81, $10 \mathrm{D} 741$ (2010)

17. W.A. Peebles. et al., Rev. Sci. Instr. 81, $10 \mathrm{D} 902$ (2010)

18. J. R. Dorris, J. C. Rost and M. Porkolab, Rev. Sci. Instrum 80, 025503 (2009) 